\title{
The Effects of Repetitive Transcranial Magnetic Stimulation Antidepressant Response on Cold Cognition: A Single-Arm Prospective Longitudinal Study
}

Helene Janine Hopman

Hiu Ying Choy

Wing Sze Ho

Hanna Lu

Wing Ho Oscar Wong

Sau Man Sandra Chan

Department of Psychiatry, The Chinese University of Hong Kong, Hong Kong, Hong Kong Special Administrative Region of China
Correspondence: Helene Janine Hopman Department of Psychiatry, The Chinese University of Hong Kong, Unit L, 19/F, Kings Wing Plaza I, 3 on Kwan Street, Shek Mun, New Territories, Hong Kong, Hong Kong Special Administrative Region of China

Tel +85255971622

Email hjhopman@cuhk.edu.hk
Purpose: The cognitive neuropsychological model of depression suggests that the cognitive deficits observed in depressed subjects are the result of attenuated top-down cognitive control resulting in increased bottom-up emotional processing. Remediation of cognitive impairments in cold cognition has been proposed as a valuable treatment for depression. The study aimed to examine the effects of clinical response to repetitive transcranial magnetic stimulation (rTMS) on cold cognition over the course of 8 weeks in medication-refractory depressed subjects.

Materials and Methods: Twenty-two medication-refractory depressed subjects received twenty sessions of high-frequency rTMS targeting the left dorsolateral prefrontal cortex, one of the key nodes of the cognitive control network. Cold cognition and antidepressant treatment response were monitored at baseline, week 2, 4 and 8. Clinical response was defined as $\geq 50 \%$ reduction in Montgomery-Åsberg Depression Rating Scale score at week 8 . Longitudinal changes in cold cognition were modeled using (generalized) linear mixed models. It was hypothesized that the excitatory effects of rTMS would improve cognition in the domains of executive function, memory, and attention. Additionally, responders were expected to show larger cognitive improvements than nonresponders.

Results: A decrease in median latency was observed on a task that measured executive function, irrespective of treatment response status. Further, responders showed significantly larger improvements in A-Prime (the ability to detect target sequences) on a sustained attention task. Post hoc analysis indicated higher levels of rumination in non-responders.

Conclusion: Our findings suggest that distractions during tasks with low perceptual complexity affected nonresponders disproportionately possibly due to higher rumination levels. Overall, cold cognition in medication-resistant depressed subjects was minimally affected by rTMS, substantiating the safety of rTMS treatment.

Limitations: The sample size was small, and the study did not include a control group.

Keywords: depression, neurostimulation, cognitive impairments, attention, executive function, memory

\section{Introduction}

Major depressive disorder is the most common psychiatric disorder and the leading cause of disability, ${ }^{1}$ affecting approximately 322 million people worldwide. ${ }^{2}$ Although the disorder is characterized by depressed mood, the role of cognitive deficits in the development and maintenance of depression pathophysiology has 
been increasingly recognized. ${ }^{3-5}$ Cognitive impairments have been associated with therapeutic response, risk of relapse, quality of life and occupational outcomes and remained, at least partially, present in remitted subjects. ${ }^{6,7}$

The cognitive neuropsychological model of depression conceptualizes cognitive deficits as two systems including cold and hot cognition that rely on distinct, but interacting brain circuits. ${ }^{4,5}$ Cold cognition refers to emotionindependent information processing that can be measured with common neuropsychological tests. A meta-analysis demonstrated impaired cold cognition in depressed subjects in the domains of executive function, working memory and attention. ${ }^{6}$ Convergent neuroimaging research has linked these impairments to abnormal activity and connectivity within the cognitive control network. ${ }^{8,9}$ Hot cognition, on the other hand, refers to emotion-laden information processing that can be measured with tasks that are reactional to emotional stimuli or contain feedback that induces a certain emotional state. ${ }^{4,5}$ Research has shown negative emotional and reward biases on tasks that rely on hot cognition in depressed subjects, ${ }^{10,11}$ which has been associated with increased activity and connectivity within the default mode network $(\mathrm{DMN})^{9,11-13}$ and between the DMN and limbic regions. ${ }^{13}$ Cold cognition can be affected by hot cognition through catastrophic responses to feedback and rumination at the cost of engaging with the external world., ${ }^{3,13}$ Additionally, due to impaired top-down control of the cognitive control network on the DMN, negatively biased thoughts have "free reign" and may self-reinforce negative cognitive schemata by primarily drawing attention to emotion-eliciting details or appraisals that are congruent with negative expectations and beliefs. ${ }^{3-5}$ In other words, the combination of attenuated top-down cognitive control and increased bottom-up emotional processing can be mutually reinforcing and perpetuate depressive symptoms. ${ }^{3}$

Based on this theoretical framework, it has been suggested that remediation of cognitive impairments may be a valuable treatment target for depression. ${ }^{4,6} \mathrm{~A}$ treatment that has shown both enhancing effects on cognition ${ }^{14,15}$ and antidepressant effects is repetitive transcranial magnetic stimulation (rTMS) to the dorsolateral prefrontal cortex. ${ }^{16}$ This non-invasive focal neurostimulation treatment has been approved by the US Food and Drug Administration for the treatment of medication-refractory depression ${ }^{17}$ and is believed to relieve core cognitive and affective symptoms by directly and indirectly targeting pathological brain networks. ${ }^{8,18}$ Although rTMS has been associated with improved working memory, ${ }^{15}$ executive function, ${ }^{19}$ attention, ${ }^{20}$ and processing speed, ${ }^{21}$ not all studies observed changes in cognition. Further, generalization of results across studies has been complicated by the large variability in stimulation protocols and cognitive tasks. ${ }^{21}$ As most studies only compared cognitive performance in one cognitive domain at two timepoints, pretreatment versus post-treatment, ${ }^{20}$ several questions remain unanswered. First, how are different cognitive domains affected by rTMS in medication-refractory depressed subjects? Second, is cognitive change a linear process? Third, are rTMS-induced cognitive changes sustainable beyond one full course of treatment?

To fill these gaps in the literature, the current singlearm prospective study examined the effects of highfrequency rTMS targeting the left dorsolateral prefrontal cortex over the course of eight weeks (baseline, week 2, $4,8)$ in the domains of executive function, memory and attention. ${ }^{6,9,15}$ Based on the literature, ${ }^{6,19,20}$ we hypothesized that stimulating the left dorsolateral prefrontal cortex would result in cognitive improvement in all tested domains due to direct excitatory effects on one of the key regions of the cognitive control network. ${ }^{22,23}$ Further, it was hypothesized that rTMS responders would show larger improvements in cold cognition over time than nonresponders because the restored ability to diminish DMN activation would indirectly improve performance due to reduced bottom-up emotional interference during cognitive tasks. ${ }^{3,4}$

\section{Materials and Methods}

\section{Participants}

Twenty-two participants that participated in another ongoing study (ClinicalTrials.gov: rTMS response trajectories in depression, NCT03348761) were approached to obtain written informed consent for participation in the current study. The study was approved by the Joint Chinese University of Hong Kong-New Territories East Cluster Clinical Research Ethics Committee and in line with the Helsinki Declaration of 1975, as revised in 2008. Participants were between 18 and 64 years old, righthanded, diagnosed with a Diagnostic and Statistical Manual of Mental Disorders - Fourth edition (DSM-IV) diagnosis of major depressive disorder, had a score of $>20$ on the Montgomery-Åsberg Depression Rating Scale (MADRS), ${ }^{24}$ had failed to respond adequately to at least one full course of antidepressant medication ( $>6$ weeks), 
were medication intolerant, or had a stable medication regime with insufficient clinical improvement. Participants were excluded from the study if they had a history of significant head trauma, neurological disorders, active abuse of alcohol or illegal substances, current psychotic symptoms, suicide ideation or recent suicide attempts, other primary DSM-IV Axis I and II psychiatric diagnoses, contraindications to magnetic resonance imaging (eg pacemaker, metal implant, pregnancy) or rTMS, or having undergone electroconvulsive therapy in the preceding year.

\section{Clinical and Outcome Measures}

Demographic and clinical characteristics were extracted from the primary study including age, gender, level of education, handedness, age at onset of first depressive episode, the total number of depressive episodes, level of treatment resistance ${ }^{25}$ and medication type. Medication did not change throughout the entire course of the study. An IQ assessment using the short form of the Wechsler Adult Intelligence Scale - Fourth Edition (Hong Kong) ${ }^{26}$ was administered by an independent psychologist to ensure similar intelligence levels for responders and nonresponders. Baseline and follow-up assessments consisted of a selected neuropsychological test battery and depressive symptom measures and were performed 7-10 days before the start of the intervention (baseline), at the end of the second week of treatment (week 2), at the end of the treatment (week 4) and four weeks post-treatment (week 8).

\section{Neuropsychological Test Battery}

Five subtests of the Cambridge Neuropsychological Test Automated Battery (CANTAB; Cambridge Cognition, Cambridge, UK) were administered by two of the authors (HYC or WSH) who were blinded to all baseline and clinical outcomes. CANTAB is a touchscreen computer-based nonverbal neuropsychological test battery that has greater precision than traditional paper-and-pencil assessments because of the automated data collection, standardized recording and scoring. The test battery was based on the meta-analytical findings from Rock and colleagues showing significant moderate cognitive deficits in executive function, memory and attention in depressed subjects compared to healthy controls (Cohen's d effect sizes ranging from -0.34 to -0.65 ). ${ }^{6}$ The battery consisted of the One Touch Stockings of Cambridge (OTS), Spatial Working Memory (SWM), Delayed Matching-to-Sample (DMS), Paired Associates Learning
(PAL), and Rapid Visual Information Processing (RVP) task. Practice effects were minimized with parallel modes and stimuli randomization within and across subjects. The entire battery was piloted in depressed subjects outside the study to ensure completion within one hour. A summary of each subtest is described below, detailed descriptions of the tasks and outcome measures can be found at https://www. cambridgecognition.com/cantab/cognitive-tests/ and Table $\mathrm{S} 1$, respectively.

\section{Executive Function \\ One Touch Stockings of Cambridge (OTS)}

OTS is a test of executive function that assessed spatial planning and working memory subdomains. Two displays containing three colored balls were presented. Participants had to move the balls in the lower display to copy the pattern in the upper display. The primary outcome measures were the number of problems solved on the first choice and the median latency to first choice.

\section{Spatial Working Memory (SWM)}

SWM is a self-ordered test that assessed the retention and manipulation of visuospatial information. The test started with colored boxes on the screen. Participants had to search for the yellow 'tokens' to fill up an empty column using a process of elimination. The outcome measure was the number of new strategies: the number of times a subject started a new search pattern from the same box as the previous trial; lower scores indicate higher strategy use.

\section{Memory Delayed Matching-to-Sample (DMS)}

The DMS is a perceptual matching and delayed visual memory test that measured visual recognition memory. Participants were presented with a target stimulus. After a short delay varying from 0,4 or 12 seconds, the target stimulus was shown along with novel alternatives. Participants were instructed to select the stimulus that matched the target stimulus. The outcome variable was the percentage of correct solutions calculated across all trials containing a delay.

\section{Paired Associates Learning (PAL)}

PAL is a test that assessed visual memory and new learning. The participants were presented with boxes, of which one or more contained a pattern. The patterns were displayed one by one in the middle of the screen and participants were instructed to point out the box where the 
pattern was originally located. As the task progressed, the number of patterns gradually increased up to eight. The total number of errors and first attempt memory score served as the main outcome parameters.

\section{Attention}

\section{Rapid Visual Information Processing (RVP)}

The RVP measured sustained attention. In the center of the screen, digits appeared (from 2-9) in random order at a rate of 100 digits per minute. Participants were instructed to detect patterns of number target sequences (eg 2-4-6) and respond as quickly as possible when the target sequence appeared by using the press pad. The primary outcome measures of this test were the median response latency on correct trials and A-Prime which is the signal detection measure of sensitivity to the target regardless of response tendency. A-Prime ranges between 0 and 1; higher scores indicate better performance.

\section{Depressive Symptom Measures}

The MADRS, ${ }^{24}$ Hamilton Depression Rating Scale, ${ }^{27}$ Clinical Global Impression scale, ${ }^{28}$ and Global Assessment of Functioning score ${ }^{29}$ were administered by a research psychiatrist (SMSC) at different time points. Further, the Chinese version of the Beck Depression Inventory II, ${ }^{30}$ a self-report questionnaire, was completed by participants. Clinical response was defined as $\geq 50 \%$ reduction in MADRS symptom scores at week 8 . The MADRS consists of ten items rated on a $0-6$ continuum $(0=$ no abnormality, $6=$ severe $)$.

\section{Intervention}

Participants received 20 sessions of neuronavigated highfrequency rTMS treatment targeting the left dorsolateral prefrontal cortex over four weeks. Individual sessions consisted of 30 minutes of $10 \mathrm{~Hz}$ rTMS (3000 pulses; 30second cycles, 5 seconds on, 25 seconds off). A Magstim Super-Rapid device was used with a 70-mm figure-ofeight double air film coil (Magstim Ltd, Whitland, UK) and manually centered at Montreal Neurological Institute coordinates $X=-46, Y=45, Z=38$ using Brainsight TMS neuronavigation (Rogue Resolutions Ltd, Montreal, Canada). Coordinates were based on a neuronavigated rTMS study by Fitzgerald and colleagues (2009) that showed enhanced response to rTMS compared to localization with the 5-cm technique. ${ }^{31,32}$ Resting motor threshold was defined as the minimum TMS intensity that elicited a motor-evoked potential of $\geq 50 \mu \mathrm{V}$ peak to peak in the contralateral abductor pollicis brevis in 5 out of 10 trials. The motor threshold was measured before the first treatment and after 10 sessions. Stimulation output was $120 \%$ of the motor threshold. Treatments were delivered by certified TMS experts (SMSC and $\mathrm{HJH}$ ).

\section{Statistical Analyses}

Longitudinal changes in the CANTAB measures were modeled using (generalized) linear mixed models ((G)LMM). Outliers were removed if the outcome values were 1.5 times the interquartile range above the upper quartile or below the lower quartile. As performances can be assumed to naturally differ between subjects during baseline, a random intercept was included. Initially, random slopes were also included to represent different temporal patterns of change in performance but subsequently discarded due to a lack of model improvement. As change is rarely truly linear, second-order polynomial terms were incorporated to capture potential non-linear changes. A stepwise approach was applied, where fixed effects were subsequently added to the model including time in weeks since baseline (4 levels: $0,2,4,8$ ), quadratic time in weeks since baseline (4 levels: $0,4,16,64)$, group ( 2 levels: nonresponders, responders), and their interactions (time $\mathrm{x}$ group, quadratic time $\mathrm{x}$ group). The analyses were controlled for variables that differed between responders and nonresponders at baseline including age and BDI baseline scores (Table 1). Categorical treatment response was defined as $\geq 50 \%$ change in MADRS symptoms score. The nonresponder group was treated as the reference group. Different models were constructed for each dependent variable: OTS median latency, OTS number of perfect solutions, SWM strategy, DMS percentage of correct solutions, PAL first attempt memory score, PAL number of errors, RVP median latency, RVP A-Prime. LMMs assuming Gaussian response were used to model changes in percentages and reaction times. GLMMs assuming Poisson response were used to model count data such as the number of errors and number of perfect solutions. ${ }^{33}$ Several parameters were used to determine the best model fit, including Akaike's (AIC) and Bayesian Information Criteria (BIC) using a smaller is better criterion, and log likelihood ratio test, which is distributed as $\chi^{2}$ with degrees of freedom equal to the number of parameters added. As maximum likelihood and restricted maximum likelihood revealed similar results, only the maximum likelihood results were reported. Structures in the residuals (ie heteroscedasticity and autocorrelation) were examined using the variance functions and correlation structures in the nlme package. ${ }^{34}$ Statistical analyses were performed by $\mathrm{HJH}$ and carried out in $R$ version 3.6.1 using the nlme (version 3.1-150), 
Table I The Means, Standard Deviations, Minimum and Maximum Values of the Demographic, Clinical and Intelligence Measures at Baseline for All Subjects and by rTMS Treatment Response Status

\begin{tabular}{|c|c|c|c|c|c|c|c|c|c|c|c|c|c|}
\hline & & & & & \multicolumn{8}{|c|}{ rTMS Response Status } & \multirow[t]{3}{*}{$p^{a}$} \\
\hline & \multicolumn{4}{|c|}{ All Subject $(n=22)$} & \multicolumn{4}{|c|}{ Responders $(n=10)$} & \multicolumn{4}{|c|}{ Nonresponders $(n=12)$} & \\
\hline & M & SD & Min & Max & $\mathbf{M}$ & SD & Min & Max & $\mathbf{M}$ & SD & Min & Max & \\
\hline \multicolumn{14}{|l|}{ Demographics } \\
\hline Gender, count F:M & \multicolumn{4}{|c|}{$17: 5$} & \multicolumn{4}{|c|}{$8: 2$} & \multicolumn{4}{|c|}{$9: 3$} & I \\
\hline Age, years & 45.23 & 10.67 & 18 & 57 & 51.60 & 4.81 & $4 I$ & 57 & 39.92 & 11.77 & 18 & 55 & $<0.01 * *$ \\
\hline Education, years & 11.48 & 3.68 & 0 & 17 & 12.44 & 3.09 & 6 & 16 & 10.75 & 4.05 & 0 & 17 & 0.29 \\
\hline \multicolumn{14}{|l|}{ Clinical characteristics } \\
\hline Age onset, years & 37.18 & 10.01 & 24 & 51 & 39.70 & 8.19 & 24 & 51 & 35.08 & 11.21 & 17 & 52 & 0.28 \\
\hline Depressive episodes, no. & 2.27 & 1.35 & I & 5 & 2.90 & 1.60 & I & 5 & 1.75 & 0.87 & I & 3 & $0.06^{+}$ \\
\hline Duration current, weeks & 38.09 & 18.64 & 4 & 52 & 30.60 & 21.15 & 4 & 52 & 44.33 & 14.29 & 12 & 52 & 0.10 \\
\hline \multicolumn{14}{|l|}{$\mathrm{TR}^{\mathrm{b}}$, count } \\
\hline Level I & \multicolumn{4}{|c|}{ I } & \multicolumn{4}{|c|}{ I } & \multicolumn{4}{|c|}{0} & 0.53 \\
\hline Level 2 & \multicolumn{4}{|c|}{16} & \multicolumn{4}{|c|}{7} & \multicolumn{4}{|c|}{9} & \\
\hline Level 3 & \multicolumn{4}{|c|}{5} & \multicolumn{4}{|c|}{2} & \multicolumn{4}{|c|}{3} & \\
\hline MADRS & 29.23 & 6.44 & 20 & 41 & 27.50 & 6.06 & 20 & 39 & 30.67 & 6.85 & 20 & 41 & 0.26 \\
\hline HAM-D & 22.55 & 7.19 & 14 & 40 & 24.60 & 6.88 & 16 & 38 & 26.33 & 7.64 & 14 & 40 & 0.58 \\
\hline CGI & $4.4 I$ & 0.67 & 3 & 5 & 4.20 & 0.63 & 3 & 5 & 4.58 & 0.67 & 3 & 5 & 0.18 \\
\hline GAF & 63.55 & 6.37 & 50 & 75 & 64.60 & 7.03 & 51 & 75 & 62.67 & 5.93 & 50 & 71 & 0.50 \\
\hline BDI-II & 36.50 & 11.76 & 16 & 56 & 29.30 & 10.66 & 16 & 52 & 42.50 & 9.20 & 25 & 56 & $<0.01 * *$ \\
\hline Intelligence & & & & & & & & & & & & & \\
\hline WAIS-IV IQ & 39.78 & 10.06 & 18 & 51 & 43.14 & 5.34 & 34 & 51 & 37.64 & 11.92 & 18 & 51 & 0.20 \\
\hline
\end{tabular}

Notes: ${ }^{\text {a The }} \mathrm{p}$-values report the significance levels reached for the t or $\chi 2$ tests (as applicable) comparing responders and nonresponders. The significance threshold was set at $\mathrm{p}<0.05$ for all comparisons. ${ }^{* *} \mathrm{p}<0.01,{ }^{+} \mathrm{p}<0.10$. ${ }^{\mathrm{b}}$ Treatment refractoriness according to the staging criteria of Thase and Rush, ${ }^{25}$ higher level indicates more refractoriness.

Abbreviations: BDI-II, Beck Depression Inventory-II; CGI, Clinical Global Impression rating scale; F, female; GAF, Global Assessment of Functioning scale; HAM-D, Hamilton Depression Rating Scale; MADRS, Montgomery-Åsberg Depression Rating Scale; M, male; TR, treatment refractoriness.

lme4 (version 1.1-25), and glmmTMB (version 1.0.2.1) packages. Since most CANTAB measures except for OTS median latency and RVP median latency have population norms, LMMs were also performed with the Z-scores for each outcome measure as outcome variable to examine whether that would influence the results. Normative Z-scores were adjusted for age, gender, and education. Given that the normative data were based on a healthy sample at a single timepoint, Cambridge Cognition discourages the use of normative data to examine change over time on their website (https:// www.cambridgecognition.com/blog/entry/research-study- design-control-groups-or-normative-data-comparison).

Nevertheless, normative data can provide information about cognitive performance at baseline compared to a non-clinical sample. Therefore, one-sample t-tests with Bonferroni correction $(p=0.05 / 6=0.008)$ for the Z-scores at baseline were performed to examine whether cognitive impairments were present at baseline.

\section{Results}

Socio-demographic, clinical and intelligence measures at baseline are summarized in Table 1. Nonresponders were 
A

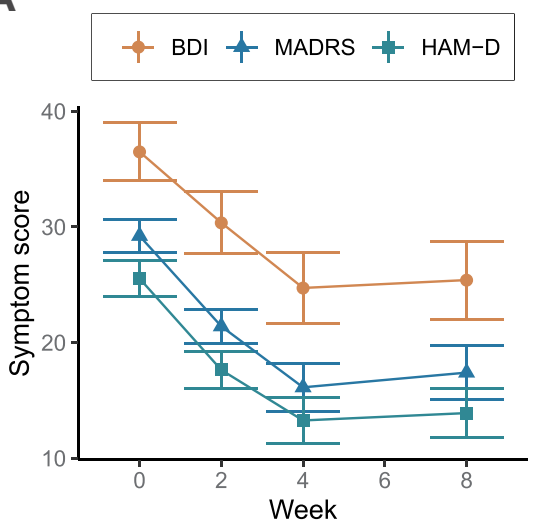

B

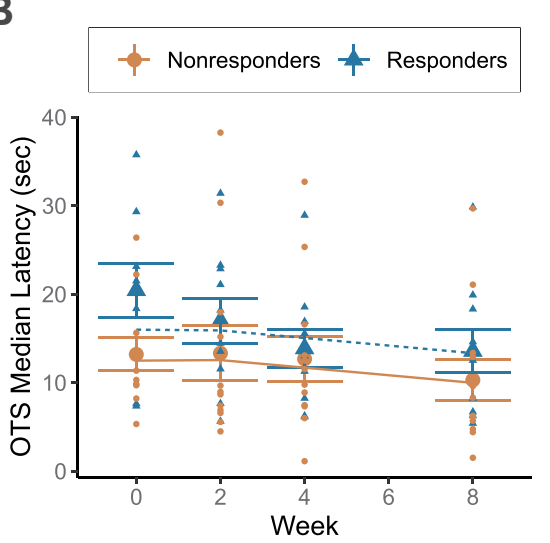

C

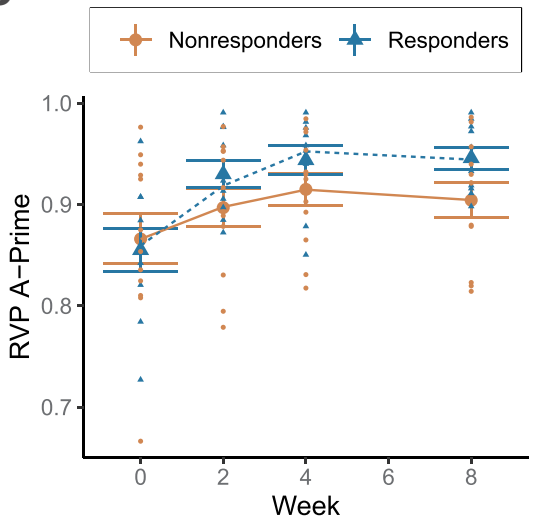

Figure I Panel (A) Line graph illustrating identical change over time for three clinical measures, including the Beck Depression Inventory-II, Montgomery-Åsberg Depression Rating Scale, and Hamilton Depression Rating Scale; symptom scores decreased until week 4 and remained fairly stable until week 8 . Panel B/C: Graphs illustrating the means, standard errors, individual datapoints and modeled trajectories for responders and nonresponders. Panel (B) shows significant linear decrease in OTS median latency over the course of 8 weeks, but no significant group differences were observed. Panel (C) shows a main effect of time and group-by-time interaction on RVP A-Prime, controlled for baseline differences in age and BDI scores, over the course of 8 weeks; responders showed significant larger improvements over time than nonresponders and performance stabilized after the last rTMS session for both groups. The clinical measures showed the reversed pattern of the RVP A-Prime trajectories, suggesting that clinical and cognitive improvement in sustained attention occurred concurrently.

significantly younger and reported higher subjective depressive scores (BDI-II) than responders at baseline. No differences were observed in gender, education, clinical and depression scores administered by a psychiatrist, and the WAIS intelligence measure. None of the subjects dropped out of the study, but a feed-forward approach was applied for one subject whose week 8 cognitive measures could not be collected. Further, missing data resulting from outlier removal varied from 0 to $11.36 \%$. Mean and standard deviations of the cognitive outcome measures are presented in the supplemental information (Table S2). Symptom score trajectories are shown in Figure 1A showing a linear decrease until week 4 which remained stable until week 8. (G)LMMs revealed no changes in performance on three of the five subtests, including SWM, DMS, and PAL (Table S3). The LMMs that modeled OTS and RVP performance over time revealed significant effects. Since the LMMs results were identical for the raw and normative Z-scores, only LMMs for the raw scores are presented.

\section{OTS}

OTS median latency to first choice was modeled with an LMM with a Gaussian distribution. Change in performance was best captured with a linear model that allowed individuals to vary randomly on the intercept. Adding the effect of group controlled for age and BDI baseline scores did not improve model fit, indicating similar baseline performance and performance trajectories in responders and nonresponders. However, adding a power variance structure reduced heteroscedasticity and improved model fit $\left(\chi^{2}(1)=32.04, p<\right.$ 0.001; Table S4). The fixed effect parameter estimates, standard errors and model summaries are presented in Table 2; the final model showed that the median latency to first choice was 14.93 seconds at baseline and decreased by 0.43 seconds per week, even after the end of the treatment $(p<0.001$, Figure 1B). Diagnostic plots indicated normality of random effects and residual errors. No significant changes over time were observed for the number of perfect solutions (Table S3).

\section{RVP}

The overall time course for change in RVP A-Prime was best captured with a second-order polynomial LMM with Gaussian distribution that allowed individuals to vary randomly on the intercept. Model comparisons showed that the effect of Group controlled for age and BDI scores on the intercept did not improve model fit $\left(\chi^{2}(3)=2.73, p=\right.$ 0.43 ), indicating no differences between rTMS responders and nonresponders in performance at baseline. However, the effect of rTMS response on both the linear and quadratic term did improve model fit $\left(\chi^{2}(5)=16.56, p<0.01\right)$, indicating that the change in RVPA A-Prime over time differed between the two groups. Adding error structures did not improve model fit (Table S4). Diagnostic plots suggest approximate normality of random effects and residual errors. Table 3 shows the fixed effect parameter estimates, standard errors and model summaries. Figure 1C illustrates the main effect of time and the group-bytime interaction, indicating that all subjects showed initial 
Table 2 Linear Mixed Model Results of the One Touch Stockings of Cambridge (OTS) Median Latency in Seconds ( $\mathrm{n}=22$, Observations $=86$ )

\begin{tabular}{|c|c|c|c|c|c|c|}
\hline & \multicolumn{6}{|c|}{ OTS Median Latency (Sec) } \\
\hline & \multicolumn{3}{|l|}{ Level-I } & \multicolumn{2}{|l|}{ Level-2 } & \multirow[b]{2}{*}{$\begin{array}{l}\text { Level-I Linear } \\
\text { VarPower=I.17 }\end{array}$} \\
\hline & Intercept & Linear & Quadratic & $\begin{array}{l}\text { Linear Group: } \\
\text { Intercept }\end{array}$ & $\begin{array}{l}\text { Linear Group: } \\
\text { Interaction }\end{array}$ & \\
\hline \multicolumn{7}{|l|}{$\begin{array}{l}\text { Fixed effects: Estimate } \\
\text { (SE) }\end{array}$} \\
\hline Intercept & $14.29(1.55)^{* * *}$ & $16.77(1.69)^{* * * *}$ & $17.56(1.81)^{* * * *}$ & I5.72 (2.43)**** & $\mid 5.04(2.51)^{* * * *}$ & $14.94(1.48)^{* * * *}$ \\
\hline Linear & - & $-0.68(0.17)^{* * *}$ & $-1.46(0.60)^{*}$ & $-0.68(0.17)^{* * *}$ & $-0.49(0.23)^{*}$ & $-0.43(0.08)^{* * *}$ \\
\hline Quadratic & - & - & $0.09(0.07)$ & - & - & - \\
\hline Age:Intercept ${ }^{a}$ & - & - & - & $2.99(1.75)$ & $2.98(1.77)$ & - \\
\hline BDI:Intercept ${ }^{\mathrm{a}}$ & - & - & - & $0.92(\mathrm{I} .1 \mathrm{I})$ & $1.53(1.77)$ & - \\
\hline Group:Intercept $\mathrm{t}^{\mathrm{b}}$ & - & - & - & $2.23(4.17)$ & $3.73(4.38)$ & - \\
\hline Group:Linear ${ }^{\mathrm{b}}$ & - & - & - & - & $-0.41(0.34)$ & - \\
\hline \multicolumn{7}{|l|}{ Model summary } \\
\hline ICC & 0.64 & & & & & \\
\hline $\mathrm{AIC}$ & 575.10 & 562.14 & 562.33 & 562.33 & 562.14 & 530.10 \\
\hline $\mathrm{BIC}$ & 582.46 & 571.96 & 574.61 & 579.51 & 582.38 & 542.37 \\
\hline Log-likelihood & -284.55 & -277.07 & -276.17 & -274.16 & -273.37 & -260.05 \\
\hline Parameters & 3 & 4 & 5 & 7 & 8 & 5 \\
\hline$\chi^{2}(\Delta d f)$ & - & $14.96 * * *$ & 1.81 & $5.81^{\mathrm{c}}$ & $7.39^{\mathrm{c}}$ & $34.04 * * * c$ \\
\hline
\end{tabular}

Notes: ${ }^{* * *} p<0.001,{ }^{*} p<0.05 .{ }^{\mathrm{a}}$ The variables age and BDI were standardized (mean $=0$, std $=1$ ). ${ }^{\mathrm{b}}$ The nonresponder group was the reference group. ${ }^{\mathrm{c}}$ Model was compared to Level-I Linear.

Abbreviations: AIC, Akaike information criterion; BIC, Bayesian information criterion; df, degrees of freedom; ICC, intraclass correlation coefficient; SE, standard error.

improvement in RVP A-Prime. However, this increase was larger for the responder group until week 4, after which RVP A-Prime stagnated until the end of the study for both groups. The clinical measures showed the reversed pattern of the RVP A-Prime trajectories, suggesting that clinical and RVP A-Prime improvement occurred concurrently (Figure 1A and $\mathrm{C}$ ). No significant changes over time were observed for RVPA Median Latency (Table S3).

\section{Normative Data}

The normative data are presented in Figure S1 and provide information about the cognitive performance relative to non-clinical norms. One-sample t-tests with Bonferroni correction indicated that two of the six measures were significantly impaired including RVP A-Prime and SWM strategy. The mean RVP A-Prime Z-score was $-0.62(S D=$ $1.03), t(21)=-2.81, p<0.01, C I=-1.08$ to -0.16 , onesided. The mean SWM strategy Z-score was $-0.40(S D=$ $0.27), t(20)=-6.71, p<0.001, C I=-0.53$ to -0.28 , onesided.

\section{Discussion}

The objective of this study was to examine the effects of rTMS clinical response on cold cognition over the course of 8 weeks across three cognitive domains including executive function, memory, and attention. The results showed that responders and nonresponders only showed different performance trajectories in the domain of sustained attention (RVP A-Prime). Furthermore, improvements in cognitive function, irrespective of responder status, were observed in the domain of executive function (OTS median latency). Finally, no cognitive deterioration and drop-outs were observed in this study, substantiating the safety and tolerability of rTMS treatment in medication-refractory depression. Here, we interpret and describe the significance of our findings.

\section{Sustained Attention}

The RVP is a serial detection task that primarily requires sustained attention, but also involves stimulus discrimination, a motor response, and recruitment of working memory processes to ensure that the correct target is identified. ${ }^{35}$ Changes over time were only observed for the A-Prime but not the median latency outcome measure, indicating changes in the ability to detect target sequences but not processing speed. The linear and quadratic effects corresponded to linear improvement during the treatment phase which stagnated during the follow-up phase. 
Table 3 Linear Mixed Model Results of the Rapid Visual Information Processing (RVP) A-Prime $(n=22$, Observations = 86)

\begin{tabular}{|c|c|c|c|c|c|}
\hline & \multicolumn{5}{|l|}{ RVP A-Prime } \\
\hline & \multicolumn{3}{|c|}{ Level-I Models } & \multicolumn{2}{|l|}{ Level-2 Models } \\
\hline & Intercept & Linear & Quadratic & $\begin{array}{l}\text { Quadratic: Group: } \\
\text { Intercept }\end{array}$ & $\begin{array}{l}\text { Quadratic: Group: } \\
\text { Interaction }\end{array}$ \\
\hline \multicolumn{6}{|c|}{ Fixed effects: Estimate (SE) } \\
\hline Intercept & $91.21(1.08)^{* * * *}$ & $89.59(1.14)^{* * *}$ & $88.22(1.17)^{* * *}$ & $88.36(1.79)^{* * *}$ & $89.72(1.84)^{* * *}$ \\
\hline Linear & - & $0.45(0.10)^{* * * *}$ & $1.79(0.33)^{* * *}$ & $1.79(0.34)^{* * * *}$ & $1.01(0.42)^{*}$ \\
\hline Quadratic & - & - & $-0.16(0.04)^{* * *}$ & $-0.16(0.04)^{* * * *}$ & $-0.10(0.05)^{*}$ \\
\hline Age:Intercept $\mathrm{t}^{\mathrm{a}}$ & - & - & - & $1.07(1.25)$ & $1.00(1.27)$ \\
\hline BDI:Intercept & - & - & - & $-1.29(1.29)$ & $-1.24(1.31)$ \\
\hline Group:Intercept ${ }^{\mathrm{b}}$ & - & - & - & $-0.32(3.05)$ & $-3.35(3.20)$ \\
\hline Group:Linear & - & - & - & - & $1.71(0.62)^{*}$ \\
\hline Group:Quadratic & - & - & - & - & $-0.14(0.07)^{+}$ \\
\hline \multicolumn{6}{|l|}{ Model summary } \\
\hline ICC & 0.70 & - & - & - & - \\
\hline AIC & -293.58 & -308.66 & -322.64 & -319.37 & -329.21 \\
\hline $\mathrm{BIC}$ & -286.22 & -298.84 & -310.37 & -299.74 & -304.67 \\
\hline Log-likelihood & 149.79 & 158.33 & 166.32 & 167.69 & 174.60 \\
\hline Parameters & 3 & 4 & 5 & 8 & 10 \\
\hline$\chi^{2}(\Delta \mathrm{df})$ & - & $17.08 * * *$ & $15.98 * * *$ & $2.73^{c}$ & $16.56 * * \mathrm{c}$ \\
\hline
\end{tabular}

Notes: ${ }^{* * *} p<0.001,{ }^{* *} p<0.01,{ }^{*} p<0.05,{ }^{+} p<0.10$. ${ }^{\mathrm{a}}$ The variables age and BDI baseline were standardized (mean $=0$, std $=1$ ). ${ }^{\mathrm{b}}$ The nonresponder group was the reference group. 'Model was compared to Level-I Quadratic. As RVPA-Prime outcomes were between 0 and I, the estimates and standard error values were multiplied by 100 to improve readability.

Abbreviations: AIC, Akaike information criterion; BIC, Bayesian information criterion; df, degrees of freedom; ICC, intraclass correlation coefficient; SE, standard error.

Further, the group-by-time interaction revealed a larger increase in A-Prime in responders than nonresponders. These results support our hypotheses in the domain of sustained attention showing 1) cognitive improvement, irrespective of rTMS response status, and 2) larger improvement in responders than nonresponders. Our findings suggest that rTMS (partially) restored the previously inefficient top-down cognitive control of the DMN, indirectly resulting in more efficient DMN suppression that allowed subjects to direct attentional resources more efficiently to external goal-directed tasks such as the RVP. ${ }^{23}$

Nevertheless, caution is required regarding the interpretation of the shape of the trajectories, because the stagnation in the responder group could also be the result of a ceiling effect as a large proportion of the responders reached A-Prime values near the maximum possible upper limit by week $4(M=0.944, S D=0.045)$. However, since nonresponders and symptom score trajectories showed similar stagnation after week 4 , improvements were presumably induced by rTMS. However, it would be interesting to examine whether larger improvements in cognition and mood could be achieved by increasing the number of treatment sessions; some patients may not have reached their full potential as the number of TMS sessions were on the lower end of the standard clinical recommendations of 20-30 sessions. ${ }^{36}$ Our findings further suggest that clinical and cognitive improvements in sustained attention occur concurrently, but unfortunately it was not possible to infer causation because measurements were not frequent enough. Finally, no changes were observed between week 4 and week 8 , indicating that the improvements last at least up to 4 weeks post-treatment. Future work should include a control group to distinguish between rTMS-induced effects and practice effects.

\section{Executive Function}

The OTS is a test of executive function which assessed spatial planning and working memory domains. The current study revealed a significant main effect of time, showing a linear decrease in median latency to first choice over the course of eight weeks for all subjects. However, the number of perfect solutions did not improve over time, indicating that subjects achieved similar accuracy levels in less time. These findings can be interpreted in two ways, either better performance due to excitatory stimulation of the cognitive control network or practice effects. Future 
work should include a healthy control group to provide a definite answer.

\section{Negative Findings}

Most of the outcome measures did not change over time. A recent meta-analysis revealed that deficits in selective attention, working memory and long-term memory persisted after subjects achieved remission and worsened with repeated episodes. ${ }^{37}$ Given that our sample showed high levels of treatment-refractoriness, we initially assumed that cognitive impairments were most likely trait markers of medication-refractory depression. However, compared to healthy normative subjects, cognitive impairments were only observed on two of the six outcome measures. There could be two explanations: 1) cognitive impairments were not present in some cognitive domains or 2) cognitive impairments could not be measured on a behavioral level. Goldstein and colleagues ${ }^{9}$ described, for example, two types of cognitive control network abnormalities in depressed subjects including hypo-activity and hyper-activity of the dorsolateral prefrontal cortex. Hypo-activity during task conditions was associated with cognitive deficits, whereas hyper-activation was interpreted as a compensation mechanism to retain normal cognitive behavior when task demands increased. Convergently, the subjects in this study were included regardless of whether the DSM-IV criterion of "a diminished ability to think or concentrate" was met. ${ }^{38}$ Although the relationship between subjective and objective cognitive impairments remains unclear, one may speculate that this somewhat conservative approach to uncover the effects of rTMS on cognition potentially resulted in an inflated type II error as improvements are more difficult to detect if cognitive deficits were not present. ${ }^{39}$ Future longitudinal controlled neuroimaging studies are needed to investigate whether minimal cognitive improvements induced by rTMS indicate trait markers, an inflated type II error due to a lack of cognitive deficits, and/or difficulties to reveal deficits due to potential neurological compensation mechanisms.

However, the question remained why an interaction effect was only observed on the RVP A-Prime outcome measure. One aspect that distinguished the RVP from the other tasks was that it required attention over a prolonged period. According to the Perceptual Load Theory, success or failure of selective and sustained attention depends on the processing demands of the task. ${ }^{40}$ Research has shown that distractions were more likely to occur during tasks with low perceptual complexity and demands, whereas distractions could be prevented by increasing the perceptual complexity of a task. ${ }^{40}$ We hypothesized that the low perceptual complexity of the RVP task could have affected nonresponders disproportionately due to a higher susceptibility to negatively biased bottom-up emotional interference and motivational deficits. ${ }^{3,4}$ Although our sample size did not allow for mediation analysis, post hoc two-way ANOVA results indeed revealed higher levels of rumination ${ }^{41}$ in nonresponders compared to responders (see supplemental information for details). Future work should further explore the potential mediation effects of rumination on tasks with low perceptual complexity compared to higher perceptual complexity to verify our hypothesis.

\section{Strengths and Confounding Factors}

One of the strengths of the current study is that cognitive performance was examined four times over a period of eight weeks using (generalized) LMMs. This method is robust to missing and unbalanced data, takes into account different spacing in time between measurements, and allows modeling of linear and nonlinear change, providing insight about 1) when changes in cognition occurred, and 2) whether change continued, stagnated or reversed after the end of a full course of rTMS treatment.

However, several confounding factors may have contributed to our findings. Responders were significantly older and reported more subjective symptoms than nonresponders. Although analyses were controlled for these differences by adding age and BDI scores at baseline to the models, complex interactions between age, subjective symptom scores, rTMS response and cognitive outcome could not be examined because of the limited sample size. Given that aging is associated with a decline in brain volume after the age of twenty with the most pronounced effects in the frontal and temporal lobes, ${ }^{42}$ it could be speculated that rTMS may affect cognition differently across the lifespan. Convergent to our findings, a deep TMS study showed better treatment response in older subjects, even though baseline cognitive performance of responders did not differ or tended to be inferior to nonresponders on several tasks. ${ }^{43}$ In contrast, a systematic review that focused on executive functions did not reveal age-related rTMS effects, ${ }^{19}$ while a recent study that examined cognitive control using a Stroop color-word interference task observed complex interaction effects. ${ }^{20}$ Accuracy improvements occurred selectively in the incongruent condition for the responder group, with the strongest benefit in older subjects. ${ }^{20}$ An interesting future direction would be to directly compare different age groups across the same neuropsychological test battery to elucidate complex interactions 
of the effects of rTMS on different cognitive domains and aging. Although a motor screening task was not part of the neuropsychological battery, we are confident that the results presented in this paper are not confounded by the effect of age on lower-order cognitive functions or familiarity with technology such as tablets because 1) the two reaction time measures (OTS and RVP median latency) did not reveal group differences, 2 ) the normative data (Z-scores adjusted for age, gender and education level) showed a similar interaction effect between time and group on the RVP A-Prime outcome variable, 3 ) if younger subjects had an advantage over older subjects because of higher familiarity with technology, better performance in younger subjects (ie the nonresponders group) would be expected while the opposite was observed; responders showed larger improvement over time than nonresponders. Finally, psychiatric medications have been associated with both cognitive impairment and cognitive improvement in depressed subjects. ${ }^{44}$ However, due to the limited sample size, the interaction between psychiatric medication and the effect of rTMS on cognition was beyond the scope of this paper. Despite this, we believe our findings are valuable because medication type and dose remained stable during the study, which mimics real-world situations where rTMS can be applied as either augmentation to ongoing pharmacotherapy or as a solitary method of treatment.

\section{Conclusion}

Clinical response to rTMS was associated with larger improvements in the domain of sustained attention. Our findings are in line with the perceptual load theory, suggesting that distractions during tasks with low perceptual complexity affected nonresponders disproportionately possibly due to higher ruminative levels. Further, no cognitive deterioration and drop-outs were observed in this study, substantiating the safety and tolerability of rTMS treatment in medication-refractory depression. Future work should increase the sample size and include a control group to elucidate complex interactions between rTMS response, age, and medication on cognition over time, and examine whether rumination levels can serve as a mediator in tasks with low perceptual complexity and demands.

\section{Data Sharing Statement}

To enhance the transparency of our research process, all data and code supporting the findings of this study are available at Mendeley Data: Hopman, Helene (2021), "Dataset: The effects of rTMS antidepressant response on cold cognition", Mendeley Data, V5, doi: 10.17632/hnxt5gzrg8.5.

\section{Acknowledgments}

This work was supported by the Research Grant Council, Hong Kong [GRF 14101714]. The funding source was not involved in the study design, data collection, data analysis, data interpretation, writing of the report, and in the decision to submit the article for publication.

\section{Author Contributions}

All authors made a significant contribution to the work reported, whether that is in the conception, study design, execution, acquisition of data, analysis and interpretation, or in all these areas; took part in drafting, revising or critically reviewing the article; gave final approval of the version to be published; have agreed on the journal to which the article has been submitted; and agree to be accountable for all aspects of the work.

\section{Disclosure}

Sau Man Sandra Chan is an editorial board member of the journal of Neuropsychiatric Disease and Treatment and reports grants from Research Grants Council, Hong Kong during the conduct of the study. The authors reported no other potential conflicts of interest for this work.

\section{References}

1. James SL, Abate D, Abate KH, et al. Global, regional, and national incidence, prevalence, and years lived with disability for 354 Diseases and Injuries for 195 countries and territories, 1990-2017: a systematic analysis for the Global Burden of Disease Study 2017. Lancet. 2018;392 (10159):1789-1858. doi:10.1016/S0140-6736(18)32279-7

2. Geneva. Depression and Other Common Mental Disorders: Global Health Estimates. Geneva; 2017.

3. Ahern E, Bockting CLH, Semkovska M, Hot-Cold Cognitive A. Model of Depression: integrating the Neuropsychological Approach Into the Cognitive Theory Framework. Clin Psychol Eur. 2019;1(3). doi:10.32872/cpe.v1i3.34396

4. Roiser JP, Sahakian BJ. Hot and cold cognition in depression. CNS Spectr. 2013;18(3):139-149. doi:10.1017/S1092852913000072

5. Roiser JP, Elliott R, Sahakian BJ. Cognitive mechanisms of treatment in depression. Neuropsychopharmacology. 2012;37(1):117-136. doi:10.1038/npp.2011.183

6. Rock PL, Roiser JP, Riedel WJ, Blackwell AD. Cognitive impairment in depression: a systematic review and meta-analysis. Psychol Med. 2014;44(10):2029-2040. doi:10.1017/S0033291713002535

7. Gonda X, Pompili M, Serafini G, Carvalho AF, Rihmer Z, Dome P. The role of cognitive dysfunction in the symptoms and remission from depression. Ann Gen Psychiatry. 2015;14(1):1-7. doi:10.1186/s12991015-0068-9

8. Liston C, Chen AC, Zebley BD, et al. Default mode network mechanisms of transcranial magnetic stimulation in depression. Biol Psychiatry. 2014;76(7):517-526. doi:10.1016/j.biopsych.2014.01.023 
9. Goldstein-Piekarski AN, Williams LM. A Neural Circuit-Based Model for Depression Anchored in a Synthesis of Insights From Functional Neuroimaging. Neurobiol Depression. 2019;241-256. doi:10.1016/B978-0-12-813333-0.00021-4

10. LeMoult J, Kircanski K, Prasad G, Gotlib IH. Negative Self-Referential Processing Predicts the Recurrence of Major Depressive Episodes. Clin Psychol Sci. 2017;5(1):174-181. doi: $10.1177 / 2167702616654898$

11. Gotlib IH, Hamilton JP, Gotlib IH, Hamilton JP. Neuroimaging and Depression Current Status and Unresolved Issues. Biol Psychiatry. 2016;17(2):159-163.

12. Li BJ, Friston K, Mody M, Wang HN, Lu HB, Hu DW. A brain network model for depression: from symptom understanding to disease intervention. CNS Neurosci Ther. 2018;24(11):1004-1019. doi:10.1111/cns. 12998

13. Kaiser RH, Andrews-Hanna JR, Wager TD, Pizzagalli DA. Large-scale network dysfunction in major depressive disorder: a meta-analysis of resting-state functional connectivity. JAMA Psychiatry. 2015;72 (6):603-611. doi:10.1001/jamapsychiatry.2015.0071

14. Kim TD, Hong G, Kim J, Yoon S. Cognitive enhancement in neurological and psychiatric disorders using transcranial magnetic stimulation (TMS): a review of modalities, potential mechanisms and future implications. Exp Neurobiol. 2019;28(1):1-16. doi:10.5607/ en.2019.28.1.1

15. Brunoni AR, Vanderhasselt MA. Working memory improvement with non-invasive brain stimulation of the dorsolateral prefrontal cortex: a systematic review and meta-analysis. Brain Cogn. 2014;86 (1):1-9. doi:10.1016/j.bandc.2014.01.008

16. O'Reardon JP, Solvason HB, Janicak PG, et al. Efficacy and Safety of Transcranial Magnetic Stimulation in the Acute Treatment of Major Depression: a Multisite Randomized Controlled Trial. Biol Psychiatry. 2007;62(11):1208-1216. doi:10.1016/j.biopsych.2007.01.018

17. Connolly KR, Helmer A, Cristancho MA, Cristancho P, O'Reardon JP. Effectiveness of transcranial magnetic stimulation in clinical practice post-FDA approval in the United States: results observed with the first 100 consecutive cases of depression at an Academic Medical Center. J Clin Psychiatry. 2012;73(4):567-573. doi: $10.4088 / \mathrm{JCP} .11 \mathrm{~m} 07413$

18. Anderson RJ, Hoy KE, Daskalakis ZJ, Fitzgerald PB. Repetitive transcranial magnetic stimulation for treatment resistant depression: re-establishing connections. Clin Neurophysiol. 2016;127 (11):3394-3405. doi:10.1016/j.clinph.2016.08.015

19. Llieva IP, Alexopoulos GS, Dubin MJ, Morimoto SS, Victoria LW, Gunning FM. Age-Related Repetitive Transcranial Magnetic Stimulation Effects on Executive Function in Depression: a Systematic Review. Am J Geriatr Psychiatry. 2018;26 (3):334-346. doi:10.1016/j.jagp.2017.09.002

20. Corlier J, Burnette E, Wilson AC, et al. Effect of repetitive transcranial magnetic stimulation (rTMS) treatment of major depressive disorder (MDD) on cognitive control. J Affect Disord. 2020;265 (June2019):272-277. doi:10.1016/j.jad.2020.01.068

21. Lantrip C, Gunning FM, Flashman L, Roth RM, Holtzheimer PE. Effects of transcranial magnetic stimulation on the cognitive control of emotion: potential antidepressant mechanisms. J ECT. 2017;33 (2):73-80. doi:10.1097/YCT.0000000000000386

22. Nejad AB, Fossati P, Lemogne C. Self-Referential Processing, Rumination, and Cortical Midline Structures in Major Depression. Front Hum Neurosci. 2013;7(October):1-9. doi:10.3389/ fnhum.2013.00666

23. Sheline YI, Barch DM, Price JL, et al. The default mode network and self-referential processes in depression. Proc Natl Acad Sci. 2009;106 (6):1942-1947. doi:10.1073/pnas.0812686106

24. Montgomery SA, Åsberg M. A new depression scale designed to be sensitive to change. Br J Psychiatry. 1979;134(4):382. doi:10.1192/ bjp.134.4.382
25. Thase ME, Rush AJ. When at first you don't succeed: sequential strategies for antidepressant nonresponders. $J$ Clin Psychiatry. 1997;58(13):23-29.

26. Wechsler D. Wechsler Adult Intelligence Scale. 4 ed. 2014.

27. Hamilton M. A rating scale for depression. J Neurol Neurosurg Psychiat. 1960;23(1):56-63. doi:10.1136/jnnp.23.1.56

28. Guy W. ECDEU Assessment Manual for Psychopharmacology. Rockville, MD: Department of Health, Education, and Welfare; 1976.

29. Del Barrio V. Diagnostic and Statistical Manual of Mental Disorders. 4th ed. Washington, DC: American Psychiatric Association Publishing; 2016. DOI:10.1016/B978-0-12-809324-5.05530-9

30. Zhang Y, Wang Y, Qian MY. Reliability and validity of the Beck Depression Inventory (BDI) examined in Chinese samples [In Chinese]. Chinese Ment Heal J. 1990;4:22-26.

31. Fitzgerald PB, Hoy KE, McQueen S, et al. A randomized trial of rTMS targeted with MRI based neuro-navigation in treatment-resistant depression. Neuropsychopharmacology. 2009;34 (5):1255-1262. doi:10.1038/npp.2008.233

32. Fox MD, Buckner RL, White MP, Greicius MD, Pascual-Leone A. Efficacy of transcranial magnetic stimulation targets for depression is related to intrinsic functional connectivity with the subgenual cingulate. Biol Psychiatry. 2012;72(7):595-603. doi:10.1016/j. biopsych.2012.04.028

33. Mirman D. Growth Curve Analysis and Visualization Using R. Boca Raton: CRC Press; 2014.

34. Pinheiro J, Bates D, DebRoy S, Sarkar D, R Core Team. \{nlme\}: linear and Nonlinear Mixed Effects Models; 2020. Available from: https://cran.r-project.org/package=nlme. Accessed April 29, 2021.

35. Neale C, Johnston P, Hughes M, Scholey A. Functional activation during the rapid visual information processing task in a middle aged cohort: an fMRI study. PLoS One. 2015;10(10):1-20. doi:10.1371/ journal.pone.0138994

36. Perera T, George MS, Grammer G, Janicak PG, Pascual-leone A, Wirecki TS. Brain Stimulation The Clinical TMS Society Consensus Review and Treatment Recommendations for TMS Therapy for Major Depressive Disorder. Brain Stimul. 2016;9(3):336-346. doi:10.1016/j.brs.2016.03.010

37. Semkovska M, Quinlivan L, O'Grady T, et al. Cognitive function following a major depressive episode: a systematic review and meta-analysis. Lancet Psychiatry. 2019;6(10):851-861. doi:10.1016/ S2215-0366(19)30291-3

38. Vinet L, Zhedanov A. A "Missing” Family of Classical Orthogonal Polynomials. 4th ed. Washington, DC: American Psychiatric Association; 2011. DOI:10.1088/1751-8113/44/8/085201

39. Wilson S, Maclean R. Research Methods and Data Analysis for Psychology. Birkshire: McGraw-Hill Education; 2011.

40. Murphy G, Groeger JA, Greene CM. Twenty years of load theoryWhere are we now, and where should we go next? Psychon Bull Rev. 2016;23(5):1316-1340. doi:10.3758/s13423-015-0982-5

41. Han X, Yang HF. Chinese Version of Nolen-Hoeksema Ruminative Responses Scale (RRS) used in 912 college students: reliability and validity. Chinese. J Clin Psychol. 2009;17(5):550-551.

42. Svennerholm L, Boström K, Jungbjer B. Changes in weight and compositions of major membrane components of human brain during the span of adult human life of Swedes. Acta Neuropathol. 1997;94 (4):345-352. doi:10.1007/s004010050717

43. Levkovitz Y, Harel EV, Roth Y, et al. Deep transcranial magnetic stimulation over the prefrontal cortex: evaluation of antidepressant and cognitive effects in depressive patients. Brain Stimul. 2009;2 (4):188-200. doi:10.1016/j.brs.2009.08.002

44. Prado CE, Watt S, Crowe SF. A meta-analysis of the effects of antidepressants on cognitive functioning in depressed and non-depressed samples. Neuropsychol Rev. 2018;28(1):32-72. doi:10.1007/s11065-018-9369-5 


\section{Publish your work in this journal}

Neuropsychiatric Disease and Treatment is an international, peerreviewed journal of clinical therapeutics and pharmacology focusing on concise rapid reporting of clinical or pre-clinical studies on a range of neuropsychiatric and neurological disorders. This journal is indexed on PubMed Central, the 'PsycINFO' database and CAS, and is the official journal of The International Neuropsychiatric Association (INA). The manuscript management system is completely online and includes a very quick and fair peer-review system, which is all easy to use. Visit http://www.dovepress.com/testimonials.php to read real quotes from published authors.

Submit your manuscript here: https://www.dovepress.com/neuropsychiatric-disease-and-treatment-journal 